\title{
Intelligent Scheduling of Smart Home Appliances Based on Demand Response Considering the Cost and Peak-to-Average Ratio in Residential Homes
}

\author{
Nedim Tutkun ${ }^{1}$, Alessandro Burgio ${ }^{2}\left(\mathbb{D}\right.$, Michal Jasinski $^{3, *}{ }^{\circledR}$, Zbigniew Leonowicz $^{3}(\mathbb{C})$ and Elzbieta Jasinska $^{4}(\mathbb{D}$ \\ 1 Department of Electrical \& Electronics Engineering, Istanbul Ticaret University, Istanbul 34840, Turkey; \\ ntutkun@ticaret.edu.tr \\ 2 Independent Researcher, 87036 Rende, Italy; alessandro.burgio.phd@gmail.com \\ 3 Faculty of Electrical Engineering, Wroclaw University of Science and Technology, 50-370 Wroclaw, Poland; \\ zbigniew.leonowicz@pwr.edu.pl \\ 4 Department of Operations Research and Business Intelligence, Wroclaw University of Science and \\ Technology, 50-370 Wroclaw, Poland; elzbieta.jasinska@pwr.edu.pl \\ * Correspondence: michal.jasinski@pwr.edu.pl
}

Citation: Tutkun, N.; Burgio, A.; Jasinski, M.; Leonowicz, Z.; Jasinska, E. Intelligent Scheduling of Smart Home Appliances Based on Demand Response Considering the Cost and Peak-to-Average Ratio in Residential Homes. Energies 2021, 14, 8510. https://doi.org/10.3390/en14248510

Academic Editor: Surender Reddy Salkuti

Received: 29 September 2021 Accepted: 13 December 2021 Published: 17 December 2021

Publisher's Note: MDPI stays neutral with regard to jurisdictional claims in published maps and institutional affiliations.

Copyright: (c) 2021 by the authors. Licensee MDPI, Basel, Switzerland. This article is an open access article distributed under the terms and conditions of the Creative Commons Attribution (CC BY) license (https:/ / creativecommons.org/licenses/by/ $4.0 /)$.

\begin{abstract}
With recent developments, smart grids assured for residential customers the opportunity to schedule smart home appliances' operation times to simultaneously reduce both the electricity bill and the PAR based on demand response, as well as increasing user comfort. It is clear that the multiobjective combinatorial optimization problem involves constraints and the consumer's preferences, and the solution to the problem is a difficult task. There have been a limited number of investigations carried out so far to solve the indicated problems using metaheuristic techniques like particle swarm optimization, mixed-integer linear programming, and the grey wolf and crow search optimization algorithms, etc. Due to the on/off control of smart home appliances, binary-coded genetic algorithms seem to be a well-fitted approach to obtain an optimal solution. It can be said that the novelty of this work is to represent the on/off state of the smart home appliance with a binary string which undergoes crossover and mutation operations during the genetic process. Because special binary numbers represent interruptible and uninterruptible smart home appliances, new types of crossover and mutation were developed to find the most convenient solutions to the problem. Although there are a few works which were carried out using the genetic algorithms, the proposed approach is rather distinct from those employed in their work. The designed genetic software runs at least ten times, and the most fitting result is taken as the optimal solution to the indicated problem; in order to ensure the optimal result, the fitness against the generation is plotted in each run, whether it is converged or not. The simulation results are significantly encouraging and meaningful to residential customers and utilities for the achievement of the goal, and they are feasible for a wide-range applications of home energy management systems.
\end{abstract}

Keywords: home energy management; binary-coded genetic algorithms; optimal power scheduling; demand response

\section{Introduction}

The gradual increase in electricity unit prices, for various reasons, brings an additional burden, especially for households. Meanwhile, technological developments in this field have brought new features to both the distribution of electrical energy and the use of electrical household appliances. This has made it possible to control electrical household appliances over the internet and to change the unit price of electricity for every hour, or even for every 15 minutes by means of a smart grid. With these developments, taking into account the usage characteristics of electrical household appliances and compromising daily usage habits, a significant reduction in daily energy costs can be achieved under the predefined constraints and conditions. This can be achieved by shifting the operating 
times of electrical household appliances to the optimal time intervals during the day, based on electricity tariffs and the avoidance of overload. There are a limited number of studies that have been conducted on this subject, and some of these studies are listed in the subsection below.

\subsection{Related Works}

Setlhaolo and Xia carried out an investigation considering the combined demand side management strategy for a limited number of houses from two aspects. The first one was the energy management system (EMS). The EMS considers demand side management strategies based on the minimization of consumer cost and the reduction of consumption from the power system, as well as $\mathrm{CO}_{2}$ emission issues through the developed model [1]. Another investigation was carried out by Shakouri and Kazemi in [2]. In the paper, in order to obtain for the household the lower energy costs, multi-objective mixed integer linear programming (MILIP) was proposed. Additionally, the validation of the proposed model based on some scenarios indicated that the reduction of daily energy costs could be on the acceptable level [2].

The authors in [3] performed the study on home energy management for residential consumers. The aim of the investigation was to decrease both their electricity bills and the peak load demand of utility companies. It was assumed that it can be achieved with a smart energy storage system (ESS) [3]. Zhao et al. were other investigators studying home EMS to schedule power consumption in households in order to obtain a reduction of electricity bills and improve the peak-to-average ratio [4]. Paterakis et al. conducted another study on the home EMS to define the optimal schedule of appliances for the day of a smart household under hourly pricing, and to reduce peak power based on demand response (DR) strategies [5]. The authors in [6] proposed a new procedure to propose the schedule of power consumption in homes equipped with ESS. In this article, the aim of power scheduling was to reduce electricity bills and improve the peak-toaverage ratio. In the investigation, the comfort of the residents was considered [6]. The authors of [7] investigated the process of the scheduling of the loads in a home EMS. This approach considers the multi-objective demand response optimization model. The indicated approach determines the scheduling of home appliances (HA) considering the non-dominated sorted genetic algorithm (GA). The authors in [8] presented a new home EMS controller. The proposed device is based on the genetic harmony search algorithm. The aim of the controller is to reduce electricity bills and the peak-to-average ratio, and to maximize user comfort. The investigated range considers a single home and multiple homes. In the investigation, real-time electricity pricing and critical peak pricing tariffs were applied. The author in [9] studied interruptible appliances (e.g., electric water heaters), although some researchers consider the problem of optimal scheduling for non-interruptible appliances; this problem was commonly formulated with integer decision variables. Zhu et al. in [10] carried out an investigation to consider the efficient solution of a complex combinatorial problem. The aim of the investigation was to define a schedule of household appliances in multiple smart homes. In the investigation, the improved cooperative heuristic approach was considered. The presented results confirmed that the proposed algorithm worked correctly. The article [11] proposed a satisfaction model for different types of household appliances. The aim of the optimization was the minimization of the energy costs. Different demand response strategies were considered e.g., demand limit based or injection limit. The article [12] presented an approach based on the fusion of the grey wolf and crow search optimization algorithm. In the investigation, the cost of electricity decrease, the extension of the users' comfort, and the minimization of the peak-to-average ratio for $\mathrm{HA}$ in the presence of real-time price signals using the indicated technique were taken into account. Adika and Wang proposed the new approach to residential customer-based demand-side management for smart charging and appliance scheduling in order to avoid overloading the power consumption, and to minimize the energy cost [11]. El-Baz and Tzscheutschler presented an algorithm that makes a simple, 
efficient, day-ahead electrical load prediction for any energy management system without requiring statistical or historical data, or using any kind of measurement sensors [12]. The authors in [13] performed an experimental study of the problem of scheduling HA from realistic points of view. Additionally, the problem of scheduling residential loads on the basis of the minimization of the cost of electricity was solved for consumer preferences [13]. Bouakkaz et al. presented a work that aims to propose the optimal strategy to schedule energy consumption to support homeowners in the reduction of energy costs, as well as saving energy in a residential home. The investigated objects were connected to a microgrid composed of a power grid system and photovoltaic and battery ESS [14]. Merdanoglu et al. investigated a model that solves the optimal scheduling problem embedded in a home EMS, enabling users to overcome the major obstacles in the implementation of DR programs. The investigation proposed the method of finding the minimum energy cost based on time-varying prices, the generation from RES, the usage demand for each HA, the battery ESS capacity and the grid constraints [15]. The authors in [16] proposed a new home energy management architecture with a renewable energy source and ESS, as well as the power grid, and in this home energy management, some mathematical models for the energy cost and peak-to-average ratio should be minimized. The applied solution is based on particle swarm optimization (PSO) and binary PSO during the day [16]. The results obtained from [16] clearly show that the proposed scheme worked well with the daily total electricity cost and the peak-to-average ratio, in comparison to those of similar works. Ahmad et al. worked to minimize the electricity bill using the scheduling of the HA and ESS in response to the dynamic pricing of the electricity market. In this study, different metaheuristic algorithms were applied, e.g., GA, binary PSO, wind-driven optimization, bacterial foraging optimization and hybrid PSO algorithms. The results corresponding to each algorithm were compared to each other through the proposed scheme [17]. It was seen that [17] is an exemplary study to test the validity of the approach and the scheme we propose compared to the studies given above; the results were produced considering the data used here, and necessary comparisons were made to obtain a better improvement in the problem of home energy management. The summary in terms of objectives, tariffs, and methods of investigated literature is given in Table 1.

Table 1. Summary in terms of objectives, tariffs, and methods of investigated literature.

\begin{tabular}{cccc}
\hline Objective & Tariff & Algorithm & References \\
\hline Cost & TOU, RTEP & MILP & {$[15]$} \\
cost \& PAR & TOU, RTEP & MILP, GA, NSGA-II, GHSA, GWCSO & {$[2-8,12,16,17]$} \\
cost \& comfort & TOU, RTEP & DA, NSGA-II, QBPSO, PSO, MILP & {$[5,9-11]$} \\
cost \& consumption & TOU, RTEP & MINLP, MILP, PSO & {$[1,13,14]$} \\
\hline
\end{tabular}

\subsection{The Original Contribution}

The aim of this study is to perform a minimization of the daily total electricity bill and the peak-to-average ratio by optimizing the start times of the shiftable smart HA on the basis of hourly day-ahead real-time electricity pricing using binary-coded genetic algorithms. The designed home energy management system includes smart HA, a power grid, a solar PV generator, and ESS; in this system, surplus energy is sold to a power grid when it is most expensive, and deficit energy is purchased from the power grid at time slots when it is least expensive if possible. There are also a few constraints in the energy storage system, in which amounts of charging and discharging are not allowed to be more than $0.3 \mathrm{kWh}$ in a single time slot. Thus, the optimization problem under consideration was solved for the optimal start times of the shiftable appliances in the home EMS using the binary-coded genetic algorithm approach. The results are compared to those obtained from few metaheuristic algorithms, and it appears that there is a considerable improvement, reducing both the electricity bill and peak-to-average ratio under similar conditions. This may be explained because, unlike the other meta-heuristic algorithms, the binary-coded genetic algorithms have more powerful genetic operators-such as selection, 
crossover, mutation, and elitism - to produce a variety of possible solutions and then refine them to reach the best possible solution over generations. It can be said that this work has contributed to this field of study, showing that binary-coded genetic algorithms with powerful genetic operators are highly efficient in improving the daily total electricity bill and peak-to-average ratio, as well as the comfort of the home.

\section{The System Architecture, Problem Definition and Applied Methods}

In this part of article, the system architecture, problem definition, and the applied approach to solve the defined optimization are detailed based on the real-time pricing scheme.

\subsection{System Architecture}

\subsubsection{Home EMS}

In general, a basic home EMS is composed of the metering infrastructure, smart metering, a home gateway, an energy management controller, smart HA, ESS, and a solar PV generator, as shown in Figure 1. In this architecture, the smart metering infrastructure covers a major role in supplying two-way communication between the smart meter and the utility for real-time electricity pricing. The smart meter installed between the infrastructure metering and the energy management controller is used to read energy consumption data, process it, and send it to the energy management controller for further handling. In this investigation, the smart household appliances are mainly divided into two groups: shiftable and non-shiftable. For instance, a washing machine or dishwasher is a shiftable home appliance, as it is operated in any time slot in the day. However, a refrigerator or lighting is a non-shiftable home appliance, because it must be operated in certain time slots during the day. Once the optimization procedure is complete, the optimal operating times of the switchable appliances are transmitted to the energy management controller for scheduling at the beginning of the day. The smart meter and the home gateway communicate with each other through $\mathrm{Wi}-\mathrm{Fi}$ in the home local area network. The energy management controller in the home gateway schedules the operation time of the appliances after receiving a real-time electricity price signal from the utility.

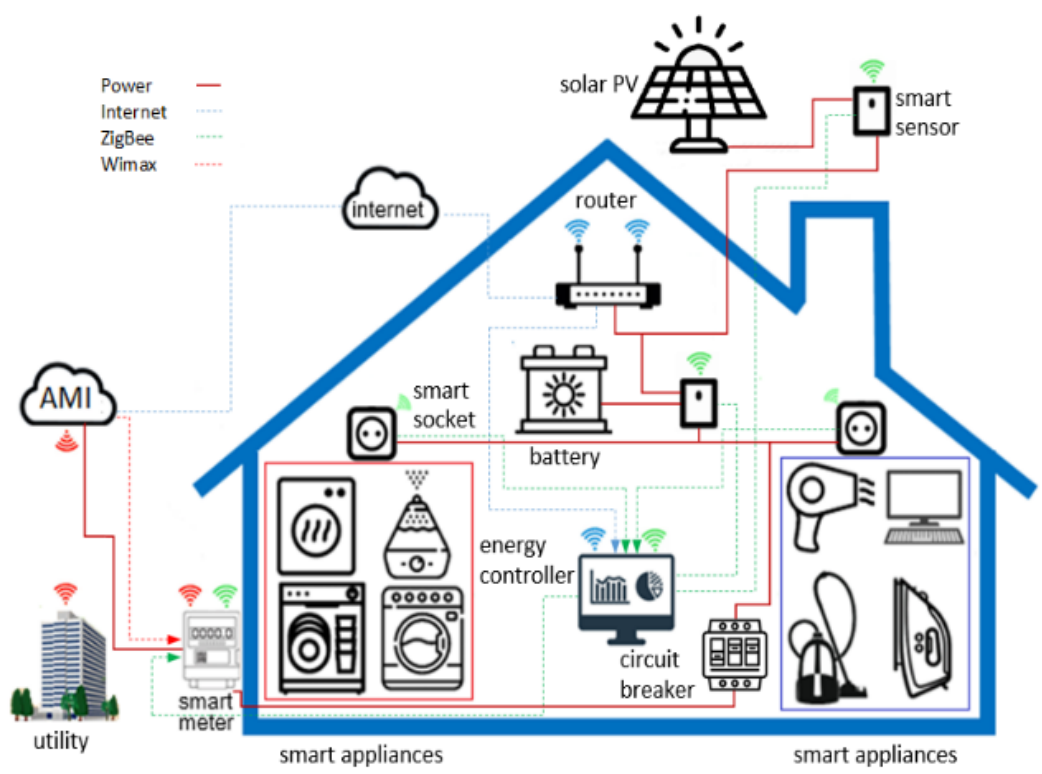

Figure 1. Basic architecture of the HEMS with a wireless home area network.

Because the first objective aims to reduce the daily electricity bill, shiftable HA-such as a washing machine, a dishwasher or a dryer for clothes-are expected to operate in the time slots when the electricity price is lowest; hence, this may lead to the overload of distribution lines and an increase in power losses beyond the typical values. However, 
householders usually prefer to operate them in the most convenient times for themselves, regardless of the maximum daily total electricity cost. For instance, the householders may intend to use the dishwasher after lunch, the washing machine in the morning, or the clothes dryer in the evening. It is obvious that there is a trade-off between the electricity cost, the householder's comfort, and the peak-to-average ratio. It is seen here that there are three objectives to be optimized, but among them the daily total electricity cost and a peak-to average ratio are more influential. Thus, it is essential to focus reasonably on these two objectives to be optimized, both in single and multiple cases. Table 2 shows the smart household appliances and their possible start- and end-time slots [17].

Table 2. Start, end and operation times of the appliances in use.

\begin{tabular}{|c|c|c|c|c|c|}
\hline Appliance & Type & Power (kW) & Length * & Start * & End * \\
\hline Washing machine & \multirow{5}{*}{ Shiftable } & 0.80 & 5 & 1 & 24 \\
\hline Air conditioner & & 1.30 & 10 & 1 & 24 \\
\hline Dryer for cloathes & & 0.70 & 4 & 1 & 24 \\
\hline Water heater & & 1.00 & 8 & 1 & 24 \\
\hline Dishwasher & & 0.20 & 3 & 1 & 24 \\
\hline Personnel computers & \multirow{6}{*}{ Non-Shiftable } & 0.20 & 18 & 7 & 24 \\
\hline Security cameras & & 0.10 & 24 & 1 & 24 \\
\hline Microwave oven & & 0.50 & 7 & 14 & 20 \\
\hline Refrigerator & & 0.90 & 20 & 3 & 22 \\
\hline TV & & 0.20 & 8 & 15 & 22 \\
\hline Lighting & & 0.10 & 6 & 17 & 22 \\
\hline
\end{tabular}

${ }^{*}$ LENGHT is the duration of the work cycle, which is the number of time slots that must be scheduled for each appliance; these slots are consecutive only if the appliance is an uninterruptible load. START is the first time slot in which the work cycle of an appliance can start; it is not possible to schedule the start before START. END is the last time slot in which the work cycle of an appliance can end; it is not possible to schedule the end after END.

\subsubsection{Rooftop PV Generator}

The generation of electricity by a photovoltaic panel is highly dependent on the irradiance around the site where the system was built. The maximum power that can be extracted from a PV panel is usually given by the manufacturer's datasheet. However, in some cases it can be changed on the basis of the ambient conditions, e.g., the irradiance level and temperature, etc. The estimated power generation by a PV system in any time instant can be determined by the I-V and P-V characteristics after a manipulation. As can be seen from Figure 1, the rooftop photovoltaic panels generate a maximum of $3 \mathrm{~kW}$ power at certain times in a day. The maximum power output of the PV is defined on the basis of the maximum power point tracking system at any time instant. The power generated by the panels is first used to partly or fully supply smart home appliances, and then to charge the ESS if there is surplus power. The battery bank stores both the surplus energy from the rooftop PV system and the cheapest energy from the utility, if it is not fully charged at the slot.

\subsubsection{ESS}

The ESS has a capacity of $3 \mathrm{kWh}$, and it is charged from the photovoltaic system when surplus energy is available, and the grid when the electricity price is the lowest in a day. The energy storage system is preferred for use when the electricity price is the most expensive, and when it is urgently needed. However, the disadvantage of the photovoltaic-energy storage system is that it has a limited lifetime and requires periodic maintenance. In this investigation, the energy storage system can be formulated as follows:

Let $B_{s}, B_{c}^{(t)}, B_{d}^{(t)}, \eta_{c}, \eta_{d}, B_{c}^{\max }$ and $B_{d}^{\min }$ be the storage capacity of the battery bank, the charging energy during the $t^{\text {th }}$ slot, the discharging energy during the $t^{\text {th }}$ slot, the charging efficiency, the discharging efficiency, the maximum energy charging level and the 
minimum energy discharging level, respectively. The state of charge of the energy storage system during the $t^{\text {th }}$ slot can be expressed as

$$
B_{b}^{(t)}=B_{b}^{(t-1)}+\eta_{c} B_{c}^{(t)}-\frac{B_{d}^{(t)}}{\eta_{d}}
$$

where $B_{b}^{(t)} \leq B_{s}$. It should be noted that the battery cannot be charged or discharged at the same slot, hence $B_{c}^{(t)}>0, B_{d}^{(t)}=0$ or $B_{c}^{(t)}=0, B_{d}^{(t)}>0$. Table 3 illustrates the characteristics of the energy storage system.

Table 3. The characteristics of the ESS in use [17].

\begin{tabular}{cc}
\hline Parameter & Value \\
\hline Roundtrip Efficiency & 0.95 \\
Charging Efficiency & 0.95 \\
Discharging Efficiency & 0.95 \\
Maximum Energy Capacity & $3 \mathrm{kWh}$ \\
Minimum Energy Capacity & $0.5 \mathrm{kWh}$ \\
Initially Stored Energy & $0.5 \mathrm{kWh}$ \\
Max. Charging/Discharging Energy & $0.3 \mathrm{kWh}$ \\
\hline
\end{tabular}

\subsection{Problem Definition}

In order to formulate the optimization problem, one day is divided into 24 equally spaced time slots, and the minimum time resolution is an hour. Let $T$ denote a set of time slots; it can be defined by

$$
T=\{1,2,3, \ldots, 120\} \forall t \in T
$$

Let $A$ denote a set of the interruptible, uninterruptible and baseline household appliances:

$$
A=\left\{a_{1}, a_{2}, a_{3}, \ldots, a_{i}, \ldots, a_{16}\right\}
$$

where $a_{i}$ is the $i^{\text {th }}$ appliance of the set.

The power consumption vector for appliance $a_{i}$ can be defined as

$$
P_{a_{i}}=\left[p_{a_{i}}^{(1)}, p_{a_{i}}^{(2)}, \ldots, p_{a_{i}}^{(t)}, \ldots, p_{a_{i}}^{(120)}\right]
$$

where $p_{a_{i}}^{(t)}$ is the average power consumption for appliance $a_{i}$ during the $t^{\text {th }}$ interval.

Let $\alpha_{a_{i}} \in T$ and $\beta_{a_{i}} \in T$ be the start and end times of the operation interval respectively, and $\alpha_{a_{i}}<\beta_{a_{i}}$ for appliance $a_{i}$. Similarly, let $l_{a_{i}}$ be the operation length of appliance $a_{i}$; thus, $l_{a_{i}}$ must satisfy the constraint $\beta_{a_{i}}-\alpha_{a_{i}} \leq l_{a_{i}}$. It should be emphasized that the larger $\beta_{a_{i}}-\alpha_{a_{i}}$, the more possible solutions to the problem. Now, we need to assume that appliance $a_{i}$ operates during $l_{a_{i}}$ without interruption. Let $s_{a_{i}}$ and $e_{a_{i}}$ be the start and end times of appliance $a_{i}$, respectively; hence $e_{a_{i}}=s_{a_{i}}+l_{a_{i}}$ and $\alpha_{a_{i}} \leq s_{a_{i}} \leq \beta_{a_{i}}-l_{a_{i}}$.

Now, the power consumption for appliance $a_{i}$ during the $t^{t h}$ interval can be expressed as

$$
P_{a_{i}}^{(t)}= \begin{cases}P_{r a_{i}}, & t \in\left[s_{a_{a}}, s_{a_{i}}+l_{a_{i}}\right] \\ 0, & t \notin\left[s_{a_{i}}, s_{a_{i}}+l_{a_{i}}\right]\end{cases}
$$

where $P_{a_{i}}$ is the average power consumption in $\mathrm{kW}$.

The total daily power consumption can be calculated by

$$
P_{\text {total }}=\sum_{t=1}^{120}\left(\left(\sum_{i=1}^{13} p_{a_{i}}^{(t)}\right)+\left(\sum_{j=1}^{5} p_{b_{j}}^{(t)}\right)\right)
$$


where $P_{b_{j}}$ is the average power consumption for the $j^{\text {th }}$ fixed time operated appliance in $\mathrm{kW}$.

Although there was a considerable improvement in the reduction of the daily cost, householder comfort inevitably decreased. Therefore, it is necessary to reduce the delay time in the scheduled operation as much as possible using the multi-objective optimization approach. The delay time for appliance $a_{i}$ can be calculated by

$$
D_{a_{i}}=\left(s_{a_{i}}\right)_{\text {sch }}-\left(s_{a_{i}}\right)_{\text {unsch }}
$$

where $\left(s_{a_{i}}\right)_{s c h}$ and $\left(s_{a_{i}}\right)_{\text {unsch }}$ are the start times for appliance $a_{i}$ in the scheduled and unscheduled operations, respectively.

If the electricity cost is the only objective, the fitness function can be expressed as

$$
f_{\text {cost }}=\operatorname{Min}\left(\sum_{t=1}^{24}\left(\left(\sum_{i=1}^{5} p_{a_{i}}^{(t)}\right)+\left(\sum_{j=1}^{6} p_{b_{j}}^{(t)}\right)\right)\right)
$$

If the delay ratio is only objective, the fitness function can be expressed as

$$
f_{\mathrm{PAR}}=\operatorname{Min}\left(\frac{\operatorname{Max}\left(\left(\sum_{i=1}^{5} p_{a_{i}}^{(t)}\right)+\left(\sum_{j=1}^{6} p_{b_{j}}^{(t)}\right)\right)}{\frac{1}{24} \sum_{t=1}^{24}\left(\left(\sum_{i=1}^{5} p_{a_{i}}^{(t)}\right)+\left(\sum_{j=1}^{6} p_{b_{j}}^{(t)}\right)\right)}\right)
$$

If the cost and the peak-to-average-ratio are the only two objectives, the fitness function can be expressed as

$$
f=\operatorname{Min}\left(\omega_{1} f_{\cos t}+\omega_{2} f_{\mathrm{PAR}}\right)
$$

where $\omega_{1}=1 / 3$ and $\omega_{2}=2 / 3$.

\subsection{Proposed Method}

The BCGA is highly fitted for solving the single objective and multi-objective optimization problems for the optimal start times of the shiftable appliances. The flowchart of BCGA method is presented in Figure 2. First, the start times of the shiftable appliances were generated by random binary strings, and their fitness values were calculated by the fitness function. The most fitted individuals were selected through the tournament selection mechanism, and the selected individuals were copied to the mating pool. A single point crossover was implemented on the selected population with a probability of 0.8 , and the inverse mutation operation was applied to the current population, with a probability of 0.1 . The elitist strategy was implemented on the mutated population to keep the best solution through the generations. The indicated process is repeated by the time the number of generation reaches the maximum number of generation, and the naturally possible solutions are improved through the successive generations, as expected. During the genetic process, the population size and the maximum number of generation were 200 and 300 , respectively. 


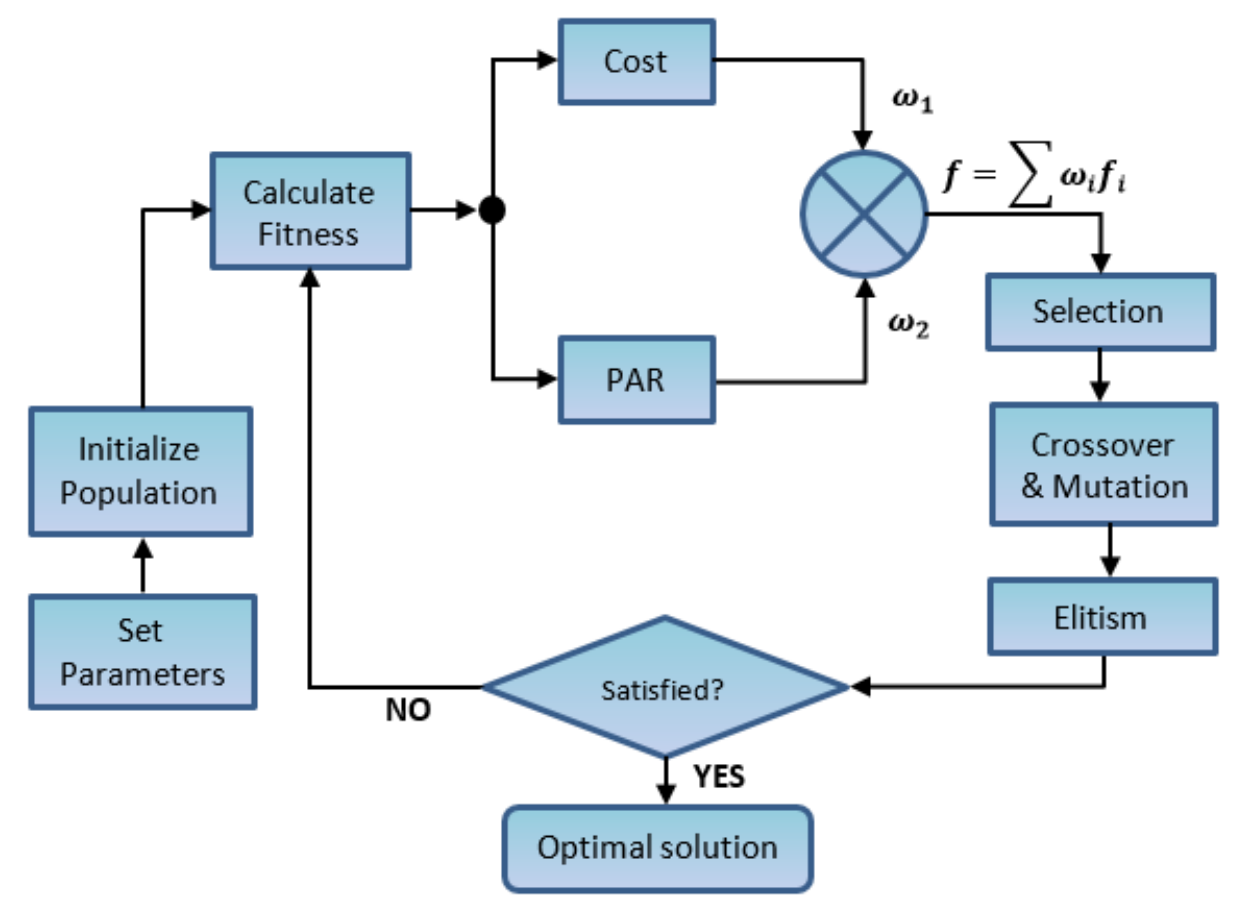

Figure 2. Flowchart of the proposed BCGA optimization.

\section{Results and Discussion}

In the above section, the simulation results obtained by the applied method are presented. This simulation includes daily energy cost minimization based on the electricity prices shown in Figure 3, after the use of electrical appliances in a typical house during the day. Divided into 24 equal time slots, the first time slot of the day indicates the 00-01-h interval, the second time slot shows the 01-02-hour interval, and so on. In this study, the optimization process includes: (I) minimizing the daily total energy cost, and (II) minimizing both the daily total energy cost and the PAR. Hence, the optimization problem was solved for an optimal solution for a single objective and two objectives within the framework of the specified conditions. As in the previous study [17], we have a $3 \mathrm{kWh}$ energy storage system and a $2 \mathrm{~kW}$-solar PV system connected to the main grid. The power demand for the smart home appliances was first met from the solar PV system; in case it was insufficient, it was met from the energy storage system. If these two are in short supply, the remaining amount is supplied from the main grid. In the case of surplus power generated by the solar PV, it is sold to the grid at the grid purchase price. The optimization problem was solved by a desktop computer with Intel (R) Core (TM) i5-10210U CPU@1.60 GHz 16.0 GB RAM in five minutes. The results obtained by using the proposed method were compared with previous studies under the same conditions, and it can be said that they were improved compared to those in [17].

In order to fairly compare the results of our proposed approach, the data and results of the previous study [17] were used, and the comparison results are given through figures and tables. In a previous study [17], electrical household appliances were divided into two groups-non-shiftable and shiftable-as shown in Table 2. As expected, when the shiftable home appliances are shifted to time slots when the electricity price is cheap, the total daily energy cost decreases along with the householder's comfort; however, this is not a desired case. Here, of course, the householder's comfort may be expected to be included in the optimization process, but this is beyond the scope of the study. The solar PV system consisting of monocrystalline panels generates a maximum power of $2 \mathrm{~kW}$ during the day, and its hourly power generation is shown in Figure 4. The energy storage system used for this study has the same physical characteristics with the one given in the previous investigation [17]. Unlike the previous study [17], the scheme we propose is flexible, and home appliances are first fed from the solar PV system in the daytime, and then from 
the energy storage system if it can provide sufficient power. The surplus energy is first evaluated in the energy storage system; the remaining energy is sold to the grid, thus reducing the cost.

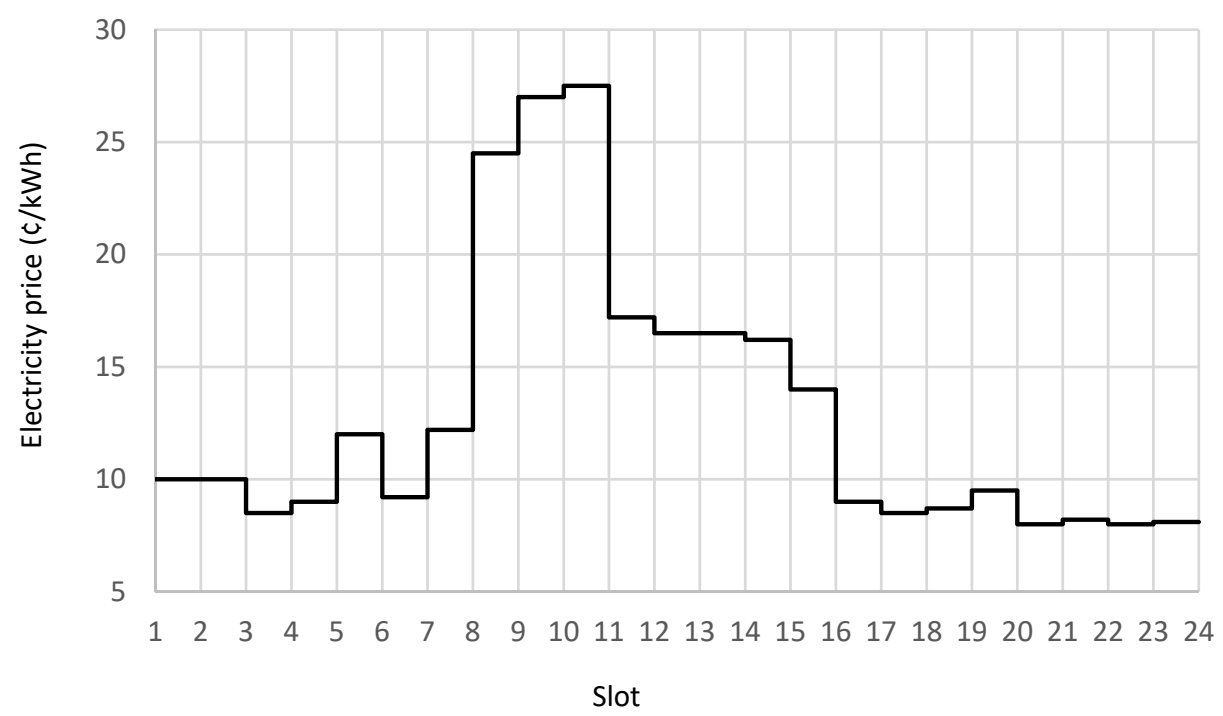

Figure 3. The hourly changing electricity prices, with the slots.

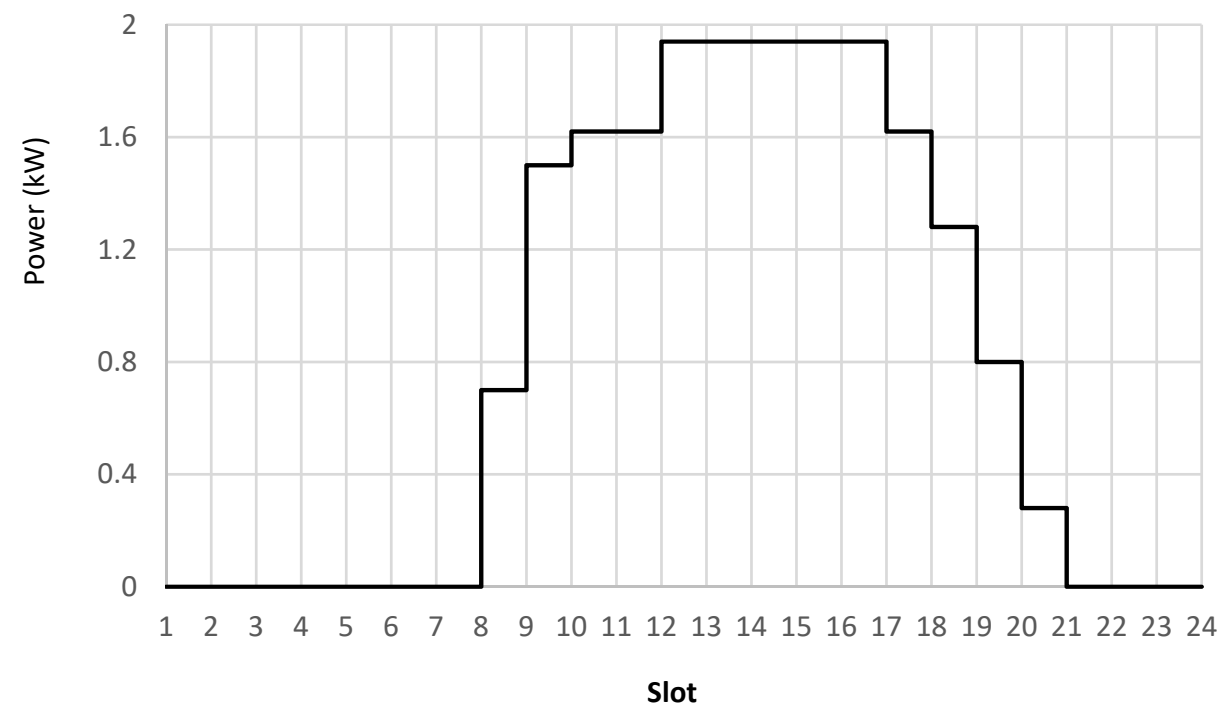

Figure 4. The hourly changing power generated by the solar PV power, with the slots.

\subsection{Single Objective Optimization}

In Equation (8), the weight numbers $\omega_{1}=1$ and $\omega_{2}=0$ are used as the objective function to minimize the total daily energy cost while calculating the optimal daily total cost, and in this calculation, the unit electricity price sold to the grid and the unit purchased from the grid are the same. The optimization process is detailed below.

Total Energy Cost

The comparison of the proposed approach with the results given in the previous study [17] for the total daily billing cost and PAR is shown in Figure 5. As seen in Figure 5a, the total cost of the daily electricity bill with the proposed method is 511.06 cents, which is better than those obtained with other methods [17]. There is an $8 \%$ reduction in the daily total electricity bill cost when the result is compared with the minimum result in previous studies [17]. As seen in Figure 5b, the PAR found is greater than only one of the results obtained from the algorithms used in the previous study [17]. However, in order 
to further improve the current results, it is necessary to solve a bi-objective optimization problem. In order to understand how this improvement was achieved, the graphs in Figure 6 should carefully be analysed. As seen in Figure 6a, there is a significant daily total cost difference between the optimized and non-optimized load dispatch. If attention is paid, in the scheduled home appliances, there is more power demand for them in the time intervals where the electricity price is lower, and less power demand in the intervals when the electricity price is higher. It can be said that this leads to a significant reduction in the daily total electricity bill. For example, while the electricity price is the lowest in the fourth time slot, the unscheduled home appliances of $1 \mathrm{~kW}$ are increased to $4 \mathrm{~kW}$ with an additional $3 \mathrm{~kW}$. The scheduled average power demand between the 8 and 11 time slots, where the electricity price is the highest, is less than the unscheduled power demand, and this plays an important role in the reduction of the total daily billing cost.

(a)

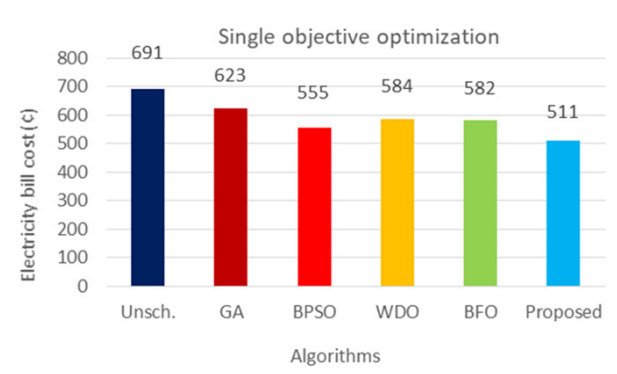

(b)

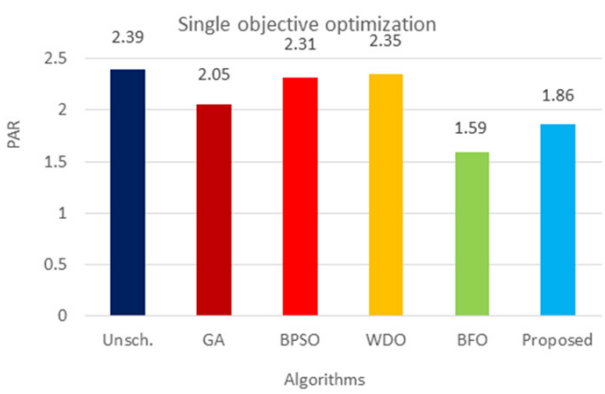

Figure 5. Comparison of the results with previous ones [17]: (a) daily total cost, and (b) PAR.

As seen in Figure 6b, while the energy received from the grid is less than others in the 8-11 time slots in the case of scheduled HA, the energy transferred from the energy storage system to home appliances is not available. Another important contribution to the reduction of the daily total electricity bill cost comes from the solar PV system; hence, this system feeds a limited number of the home appliances in times when electricity prices are high, leading to a decrease in the daily total electricity bill cost. In addition, it causes the daily total cost to decrease by transferring the surplus energy it produces to the ESS and the grid. However, some energy loss during the charging and discharging of the energy storage system from the solar PV system reduces the total efficiency of the entire system by $5 \%$. In this regard, it is preferable to use the power obtained from the solar PV system to directly feed the household appliances. The operation times of the shiftable household appliances optimized in the performed simulation are given in Table 4.

Table 4. The optimized operation times of the appliances in SOO.

\begin{tabular}{cccc}
\hline Type & Appliance & OT & SOO \\
\hline \multirow{3}{*}{ Shiftable } & Washing machine & 5 & 14 \\
& Air conditioner & 10 & 3 \\
& Dryer for clothes & 4 & 19 \\
& Water heater & 8 & 3 \\
& Dishwasher & 3 & 3 \\
\hline
\end{tabular}




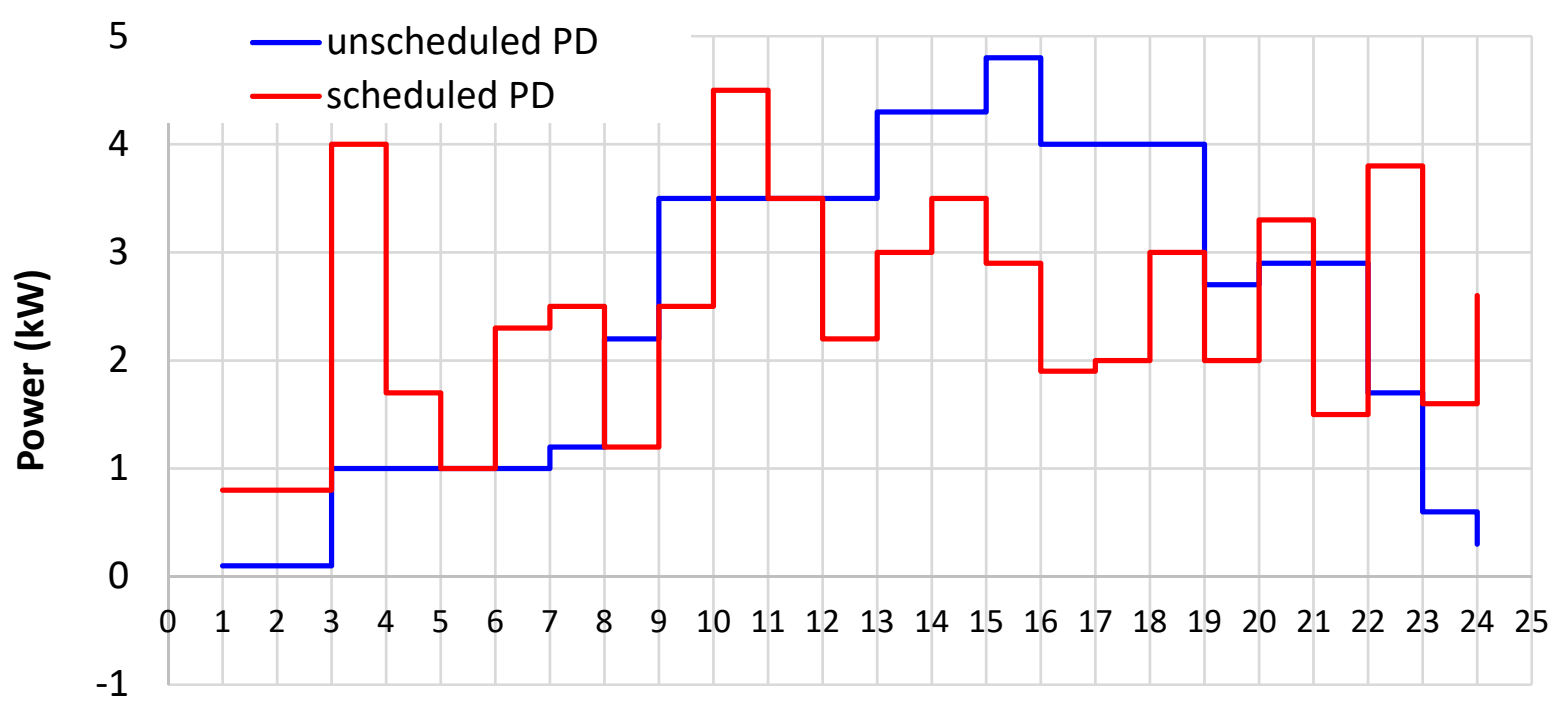

Slot

(a)

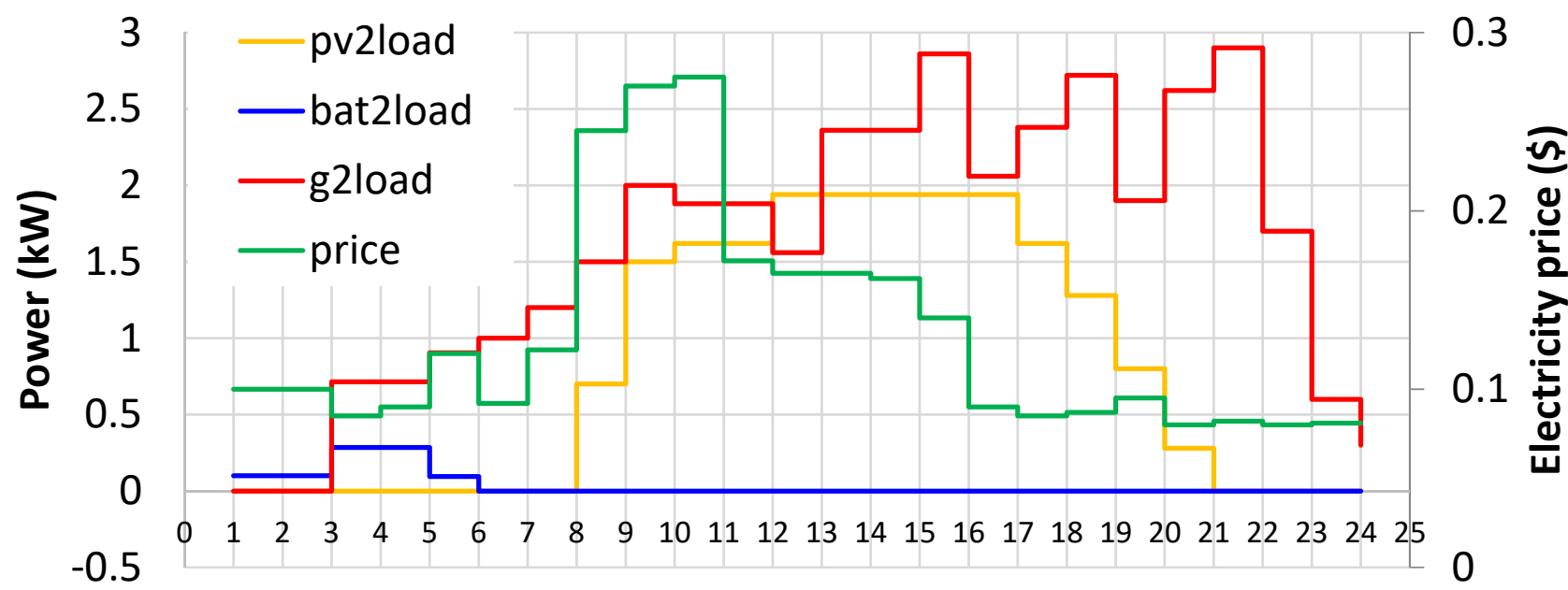

Slot

(b)

Figure 6. (a) The scheduled and unscheduled daily power demand, and (b) the powers delivered to the load. pv2load, PV to load; bat2load, battery to load; g2load, grid to load; price, electricity price in $\$$.

\subsection{Bi-Objective Optimization}

The PAR is an important indicator of the behaviour of the householder's home appliances and significantly affects the performance of the main grid. The PAR rises as expected when we only focus on minimizing the daily total electricity bill cost. In order to overcome this problem, it is necessary to optimize both the daily total electricity bill cost and the PAR at the same time. It is obvious that the problem in question is a bi-objective optimization problem, and the two objective functions are converted to a single objective function with the help of constant weight coefficients. The selection of the coefficients is made according to the principle of the equalization of the maximum values; hence, the bi-objective functions take in a certain interval, with their sum being equal to unity. In the optimization process, the input values related to the method used for single optimization are taken as they are, 
except for the fitness function. The simulation time slightly increases because the problem is a little more complex than the single optimization problem.

While the simulation results illustrated in Figure 7 produce better results compared to the results obtained from the previously used methods [17], a better improvement was achieved in the result obtained in the single optimization. As can be seen from Figure 7 , different weight coefficients produce slightly different results, and $w_{1}=1 / 3$ and $w_{2}=2 / 3$ yielded the best results among the three selected weight coefficients of $w_{1}=1 / 2, w_{2}=1 / 2$, $w_{1}=2 / 3$ and $w_{2}=1 / 3$. Contrary to what is expected here, in all three cases, the total daily cost has almost no change at all, and is very close to the result of the single optimization. The shiftable optimal operation hours obtained by bi-objective optimization by selecting appropriate weight coefficients are given in Table 5. As seen in Table 5, while the PAR is significantly reduced, the starting times of the shiftable household appliances are different from those obtained by single-objective optimization, and the best result was found when the weight numbers were $\omega_{1}=1 / 3$ and $\omega_{2}=2 / 3$.

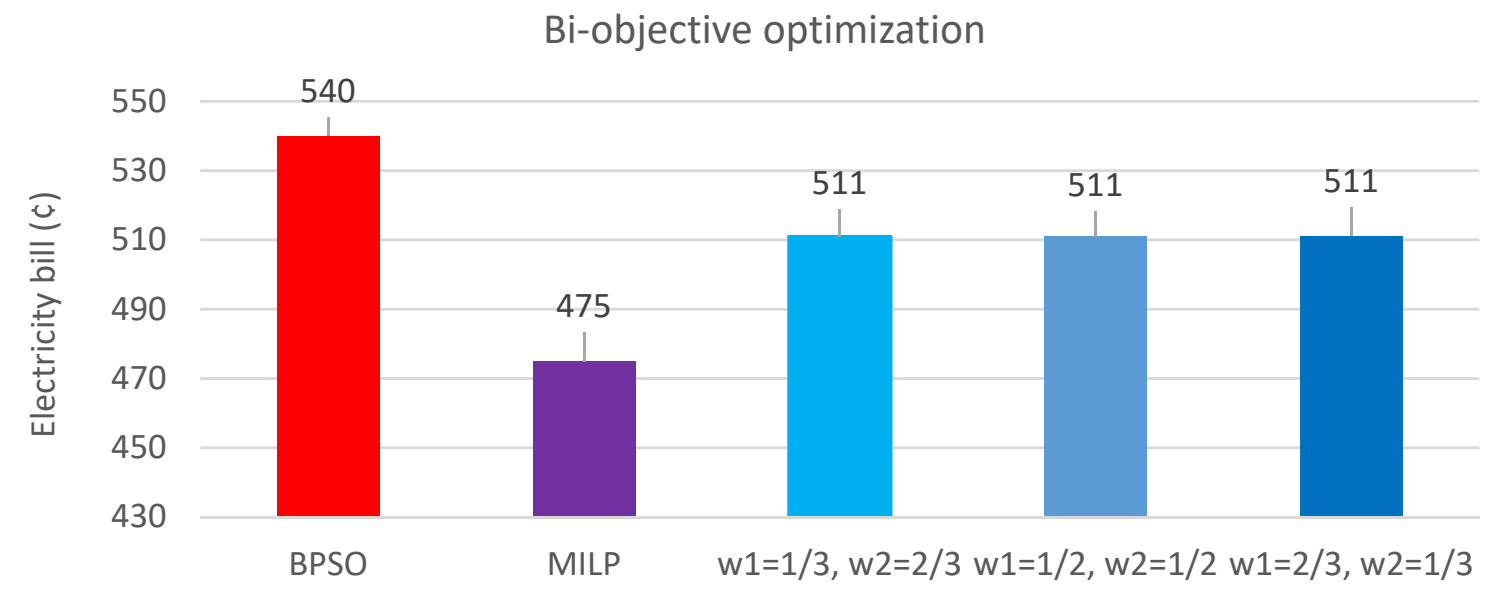

Algorithms

(a)

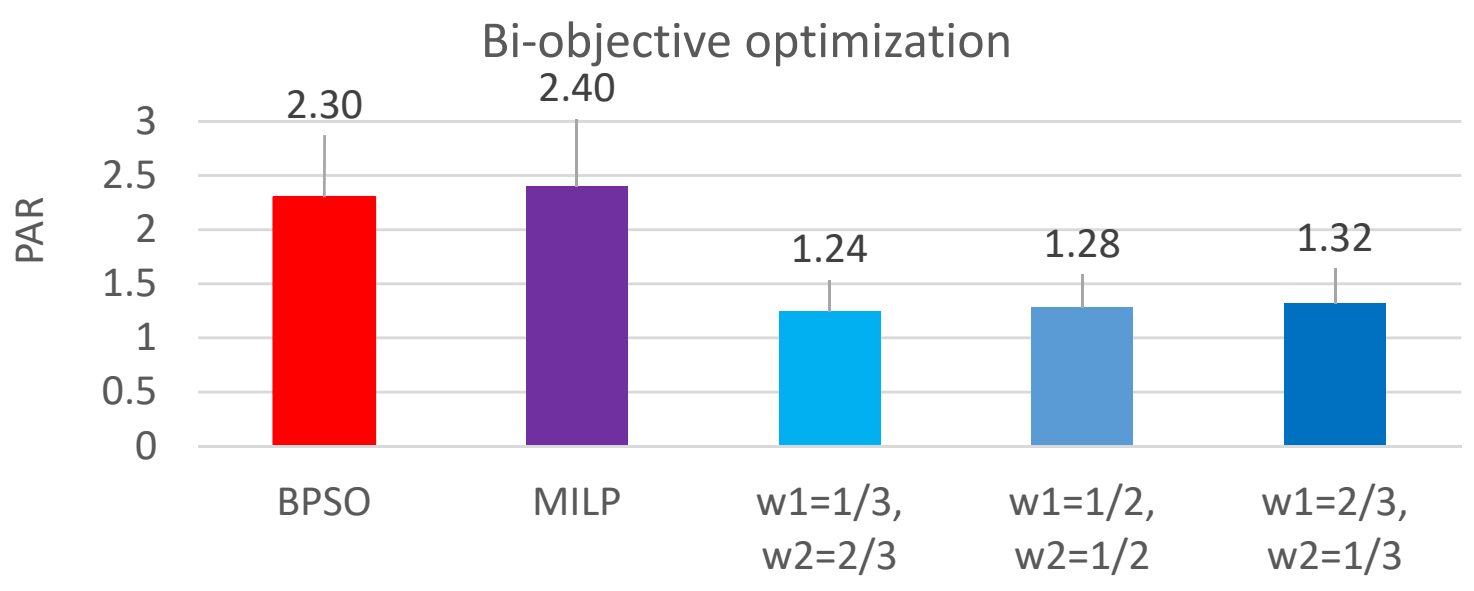

Algorithms

(b)

Figure 7. The comparison of the results with the previous ones [17]: (a) daily total cost, and (b) PAR. 
Table 5. The optimized operation times of the appliances in BOO.

\begin{tabular}{cccc}
\hline Type & Appliance & OT & BOO \\
\hline \multirow{3}{*}{ Shiftable } & Washing machine & 5 & 7 \\
& Air conditioner & 10 & 1 \\
& Dryer for clothes & 4 & 12 \\
& Water heater & 8 & 1 \\
& Dishwasher & 3 & 1 \\
\hline
\end{tabular}

In the designed bi-objective optimization, the PAR was reduced from 1.859 to 1.24; therefore, this is a very good improvement on the PAR drop. However, in this optimization process, the daily total electricity cost surprisingly remained constant at 511 US cents for three different weight coefficient sets. In the previous study [17], the cost of 475 cents obtained from the use of the mixed-integer linear programing (MILP) seemed to be a nonfeasible solution, such that if all appliances are operated at the time slot when the electricity price is the lowest, their daily total cost is 464.80 US cents; this is really nonsense. Actually, this verifies that a PAR of 2.4 indicates that the household appliances are overlapped in a certain time slot. The variation of the hourly power demand of household appliances with the time slots and powers delivered to these appliances are shown in Figure 8. It can be seen from Figure 8a that the largest power demanded from the main grid is 4.5 $\mathrm{kW}$ in the 11th time period, and the large power demands are spread over low-priced time slots. Although this is the most influential factor that helps to significantly reduce the large PAR values, the additional power boost from the solar PV system at noon has a significant share in this decrease. As can be seen from Figure $8 \mathrm{~b}$, the reduction in the daily total cost is intended to meet the power needed by the appliances when the electricity price is the lowest. Note that here, the power transferred from the energy storage system to the appliances is not sufficient, as expected. The power boost from the solar PV generator appears to be effective at between 09 and $18 \mathrm{~h}$, in contrast to the energy storage system in use.

In the light of all of these results, it can be seen that the proposed method, due to its nature, produces better results in terms of applicability when compared to other methods for the reduction of the daily total cost and PAR in the home energy management system. A one-to-one comparison with the results of a similar study was clearly demonstrated here. 


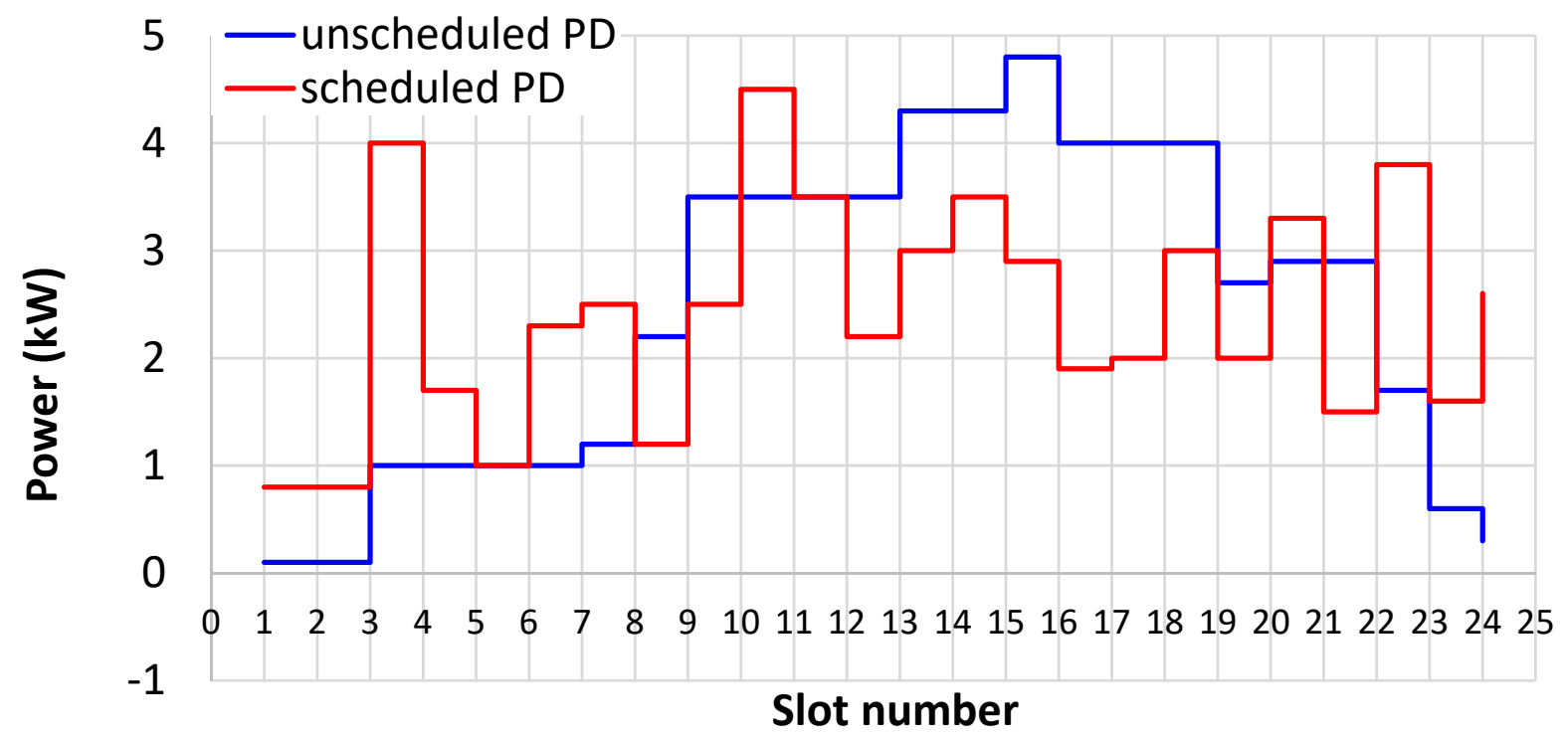

(a)

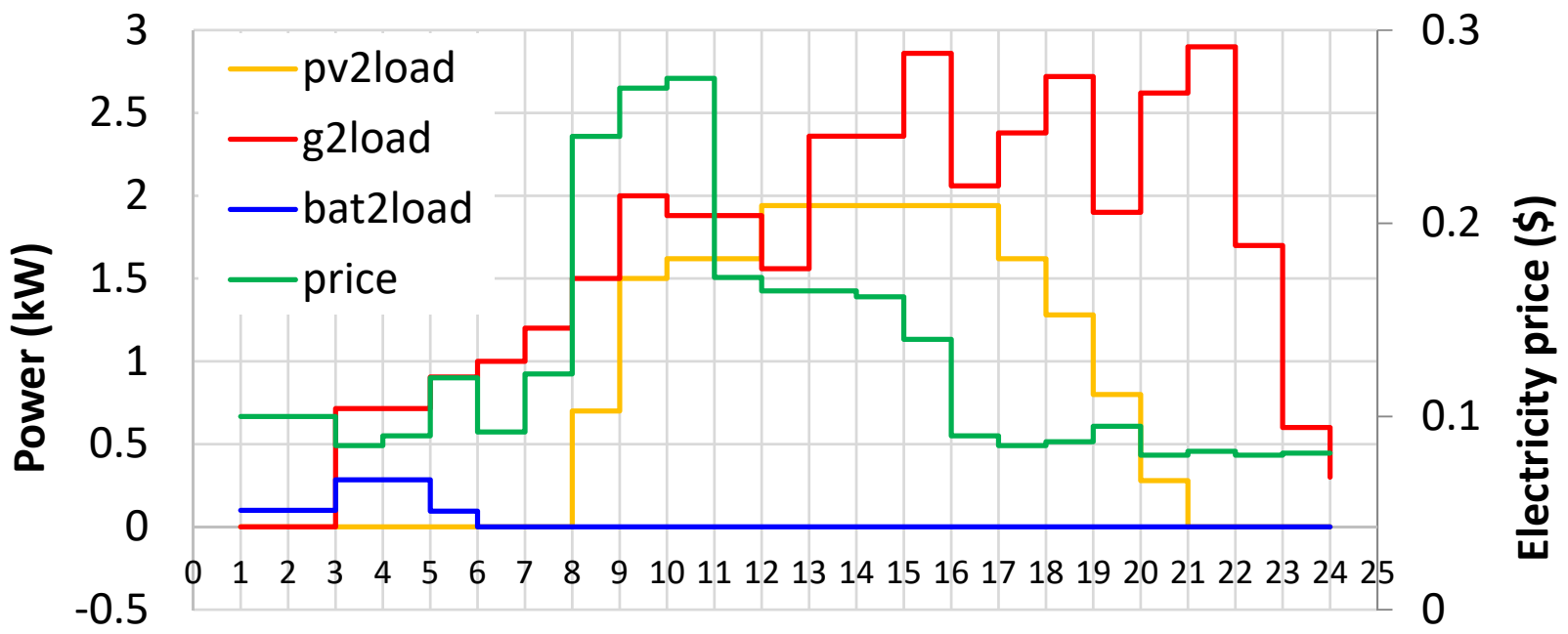

Slot

(b)

Figure 8. (a) The scheduled and unscheduled daily power demand, and (b) the powers delivered to the load.pv2load, PV to load; bat2load, battery to load; g2load, grid to load; price, electricity price in $\$$.

\section{Conclusions}

Scheduling smart home appliances' operation times to simultaneously reduce both the electricity bill and the PAR based on demand response, as well as increasing user comfort, is an essential task. It is worth noting that in the scheduled operation, the cost is always lower than in the unscheduled operation. The cost value varies depending on the price of electricity, the rated power and number of household appliances, as well as the length of the operation range. Although it is desirable for the user to reduce the cost, this inevitably leads to a PAR increase in certain time intervals. This is not welcome for either the user or the utility, as it causes extra losses in power transmission lines. A gradual increase in the electricity price depending on the consumed power up to a certain level seemed to be a solution in the previous application, and it can be seen from the results that it is ineffective to decrease PAR. Instead, it was observed that the simultaneous optimization 
of both the cost and PAR offers better results to the householders. Taking user comfort into consideration, it was observed that as the user comfort increases, the PAR increases together with the cost when optimizing the cost, the PAR and the comfort simultaneously.

Author Contributions: Conceptualization, N.T.; methodology, N.T. and A.B.; software, N.T.; validation, M.J. and Z.L.; formal analysis, M.J. and E.J.; investigation, A.B.; writing-original draft preparation, N.T.; writing-review and editing, A.B.; visualization and supervision, Z.L.; project administration, N.T. and A.B.; funding acquisition, E.J. and Z.L. All authors have read and agreed to the published version of the manuscript.

Funding: This research was funded by Wroclaw University of Science and Technology, K38W05D02.

Institutional Review Board Statement: Not applicable.

Informed Consent Statement: Not applicable.

Data Availability Statement: The applied manuscript data are available on request from ntutkun@ticaret.edu.tr.

Conflicts of Interest: The authors declare no conflict of interests.

\section{References}

1. Setlhaolo, D.; Xia, X. Combined residential demand side management strategies with coordination and economic analysis. Electr. Power Energy Syst. 2016, 79, 150-160. [CrossRef]

2. Shakouri, H.; Kazemi, A. Multi-objective cost-load optimization for demand side management of a residential area in smart grids. Sustain. Cities Soc. 2017, 32, 171-180. [CrossRef]

3. Lokeshgupta, B.; Sivasubramani, S. Multi-objective home energy management with battery energy storage systems. Sustain Cities Soc. 2019, 47, 101458. [CrossRef]

4. Zhao, Z.; Lee, W.C.; Shin, Y.; Song, K.B. An optimal power scheduling method for demand response in home energy management system. IEEE Trans. Smart Grid 2013, 4, 1391-1400. [CrossRef]

5. Hussain, I.; Ullah, M.; Ullah, I.; Bibi, A.; Naeem, M.; Singh, M.; Singh, D. Optimizing energy consumption in the home energy management system via a bio-inspired dragonfly algorithm and the genetic algorithm. Electronics 2020, 9, 406. [CrossRef]

6. Sharifi, A.H.; Maghouli, P. Energy management of smart homes equipped with energy storage systems considering the PAR index based on real-time pricing. Sustain. Cities Soc. 2019, 45, 579-587. [CrossRef]

7. Veras, J.M.; Silva, I.R.S.; Pinheiro, P.R.; Rabêlo, R.A.L.; Veloso, A.F.S.; Borges, F.A.S.; Rodrigues, J.J.P.C. A multi-objective demand response optimization model for scheduling loads in a home energy management system. Sensors 2018, 18, 3207. [CrossRef] [PubMed]

8. Hussain, H.M.; Javaid, N.; Iqbal, S.; Hasan, Q.U.; Aurangzeb, K.; Alhussein, M. An efficient demand side management system with a new optimized home energy management controller in smart grid. Energies 2018, 11, 190. [CrossRef]

9. Jordehi, A.R. Binary particle swarm optimisation with quadratic transfer function: A new binary optimisation algorithm for optimal scheduling of appliances in smart homes. Appl. Soft Comput. J. 2019, 78, 465-480. [CrossRef]

10. Zhu, J.; Lin, Y.; Lei, W.; Liu, Y.; Tao, M. Optimal household appliances scheduling of multiple smart homes using an improved cooperative algorithm. Energy 2019, 171, 944-955. [CrossRef]

11. Liu, Y.; Xiao, L.; Yao, G.; Bu, S. Pricing-based demand response for a smart home with various types of household appliances considering customer satisfaction. IEEE Access 2019, 7, 86463-86472. [CrossRef]

12. Waseem, M.; Lin, Z.; Liu, S.; Sajjad, I.A.; Aziz, T. Optimal GWCSO-based home appliances scheduling for demand response considering end-users comfort. Electr. Power Syst. Res. 2020, 187, 106477. [CrossRef]

13. Yahia, Z.; Pradhan, A. Multi-objective optimization of household appliance scheduling problem considering consumer preference and peak load reduction. Sustain. Cities Soc. 2020, 55, 102058. [CrossRef]

14. Bouakkaz, A.; Mena, A.J.G.; Haddad, S.; Ferrari, M.L. Efficient energy scheduling considering cost reduction and energy saving in hybrid energy system with energy storage. J. Energy Storage 2021, 33, 101887. [CrossRef]

15. Merdanoğlu, H.; Yakıc1, E.; Doğan, O.T.; Duran, S.; Karatas, M. Finding optimal schedules in a home energy management system. Optim. Eng. 2020, 182, 106229. [CrossRef]

16. Mouassa, S.; Bouktir, T.; Jurado, F. Scheduling of smart home appliances for optimal energy management in smart grid using Harris-hawks optimization algorithm. Optim. Eng. 2021, 22, 1625-1652. [CrossRef]

17. Ahmad, A.; Khan, A.; Javaid, N.; Hussain, H.M.; Abdul, W.; Almogren, A.; Alamri, A.; Niaz, I.A. An optimized home energy management system with integrated renewable energy and storage resources. Energies 2017, 10, 549. [CrossRef] 